\title{
Une rencontre épistémologique
}

\section{Introduction pédagogique et ludique à la recherche qualitative}

\section{An epistemological encounter}

\section{Pedagogical and playful introduction to qualitative research}

\author{
Marie-Pierre CODSI* \\ Université de Montréal, Montréal, Canada
}

Manuscrit reçu le 3 juin 2017 ; accepté pour publication le 12 novembre 2017

\begin{abstract}
Résumé - Contexte : L'introduction à la recherche qualitative est souvent complexe et déconcertante pour le néophyte, particulièrement dans le milieu médical où la recherche quantitative domine largement. L'auteur témoigne de cette confrontation d'idées à travers un dialogue épistémologique imaginaire. But : Présenter de façon pédagogique et ludique la pertinence et la légitimité de la recherche qualitative. Méthode: Débat épistémologique prenant la forme d'un dialogue fictif entre un médecin et une anthropologue. Résultats : Les principaux arguments et critiques attachés à la recherche qualitative sont présentés et argumentés. Conclusion : Cet essai se veut un outil pédagogique utile et pertinent pour introduire aux lecteurs les grands concepts sous-tendant la recherche qualitative. La forme simple et ludique s'adresse autant au néophyte qu'au chercheur plus expérimenté.
\end{abstract}

Mots clés : recherche qualitative, pédagogie médicale, éducation, épistémologie

\begin{abstract}
Context: In the medical field, where quantitative methods dominate research programs, qualitative research can seem overly complex and disconcerting to the neophyte. The author recognizes the differences between both research paradigms, and addresses this clash of ideas through an imaginary epistemological dialogue. Objective: To introduce in a narrative but pedagogical essay the relevance and legitimacy of qualitative research. Method: Redaction of an epistemological debate taking the form of a fictitious dialogue between a medical doctor and an anthropologist. Results: Presentation of the main supporting arguments and criticisms typically aimed at qualitative research. Conclusion: This essay is intended to be a useful and relevant educational tool used to introduce readers to the main characteristics of qualitative research. The simple and narrative form is used to target a wide audience, both neophytes and experienced researchers.
\end{abstract}

Keywords: qualitative research, epistemology, medical education

\section{Avant-propos}

Nous rapportons ici le dialogue imaginaire entre deux amis de longue date, Georges et Lucy, qui se rencontrent pour prendre un verre.

Georges Goodwill est âgé de 70 ans. Il est médecin anatomiste depuis plus de quarante-cinq ans, très connu dans son domaine pour ses nombreux travaux de recherche sur les protéines ribosomales A145. Il est un fervent défenseur du positivisme et de la recherche quantitative.

\footnotetext{
${ }^{*}$ Correspondance et offprints : Marie-Pierre CODSI, 3706 rue Saint-Hubert, Montréal, H2L 4A2 (Québec), Canada.

Mailto: marie-pierre.codsi@umontreal.ca
}

Lucy De Groot est âgée de 68 ans. Ethnologue de grand renom, elle est reconnue pour ses études sur les phonèmes des peuples Khoisan d'Afrique du Sud. Elle a une longue expérience de recherche qualitative.

Le souper prend une tournure inattendue lorsque le Dr Goodwill remet en question la pertinence et la légitimité de la recherche qualitative. S'ouvre alors un débat épistémologique unique qui introduit les grands concepts soustendant la recherche qualitative.

\section{Le dialogue}

La rencontre se déroule à une époque inconnue. Après un bon repas et quelques verres de vin, le Docteur Goodwill s'avança sur sa chaise. 
G. Goodwill : Ma chère, je suis vraiment très heureux de vous revoir; il y a longtemps que je veux vous entretenir d'un sujet particulier.

L. De Groot : Mais je vous écoute, cher ami.

G. Goodwill : Avez-vous déjà entendu parler de cette nouvelle pseudo-mode en anthropologie qui consiste à faire de la recherche dite qualitative?

L. De Groot: Mais bien sûr! J'ai utilisé cette méthode pour tous mes derniers travaux de recherche.

G. Goodwill : Comment! Vous?! Vraiment? Ce que j'avais entendu est donc vrai? s'exclama le Dr Goodwill. Ma pauvre amie, n'avez-vous donc pas peur d'être ridiculisée par la communauté scientifique? Vous qui êtes si respectée!

L. De Groot: Pourquoi devrais-je craindre d'être ridiculisée? Ces méthodes valent bien les autres... lui répondit Mme De Groot, un léger sourire malin se dessinant sur ses lèvres.

G. Goodwill : Eh bien voyons, ma chère! Vous savez comme moi que ces méthodes n'ont rien à voir avec les méthodes de recherche quantitatives, qui ont fait leur preuve depuis de nombreuses années. Tout le reste n'est que pseudo-science... La recherche doit servir, par des méthodes rigoureuses et éprouvées, à trouver la vérité, par des faits vérifiables. Vos méthodes sont remplies de biais, tous plus époustouflants les uns que les autres. Et si, malgré cela, vous arrivez à énoncer des conclusions potables, que peut-on bien dire de tout cela, voulez-vous me dire?

Lucy de Groot s'esclaffa de rire.

L. De Groot : Écoutez cher ami : si vous êtes d'accord pour que notre souper se prolonge un peu plus longtemps que prévu, je suis prête à répondre à chacune de vos critiques avec plaisir.

Georges Goodwill regarda sa compagne pendant quelques instants en silence. Puis, il appela le serveur et commanda deux Brandy.

G. Goodwill: Je vous écoute. Cependant, je vous préviens, je ne vous ferai pas de cadeaux.

L. De Groot : Je n'attends rien de moins de vous, mon cher..., répondit Mme De Groot en souriant. Alors, si vous permettez, je tenterai de répondre à vos questionnements point par point. Vous avez mentionné tout d'abord ce concept de «Vérité». Je commencerai donc à débattre avec vous sur le plan épistémologique de la recherche et de l'analyse qualitative. Puis, j'aborderai ces fameux biais qui semblent tant vous irriter. Pour finir, je pourrai conclure avec la pertinence et l'utilité de cette méthode. Cela vous convient-il?

G. Goodwill : C'est parfait, je suis impatient de vous entendre...

L. De Groot: Commençons d'abord par le premier point que vous avez soulevé, c'est-à-dire la recherche de la Vérité pour les besoins de la Science. En fait, ce que vous soulevez ici est une question de nature épistémologique. C'est une question centrale que vous posez et qui est essentielle afin de poursuivre notre discussion. En fait, ce que vous décrivez ici pose comme prémisse de base le paradigme positiviste, n'est-ce pas?
G. Goodwill : Vous parlez ici un langage qui ne m'est pas trop familier, je l'avoue.

L. De Groot: Bon, je vous propose alors de commencer par une description de ce paradigme positiviste. Ensuite, je vous présenterai les différentes critiques qui ont ébranlé cette philosophie, et qui ont ensuite permis la naissance du paradigme constructiviste/interprétatif.

Le paradigme positiviste a été à la base des fondements des sciences naturelles actuelles, grandement influencés par les célèbres penseurs des derniers siècles (Aristote, Locke, Wolff, Hume, Kant, etc.). Reposant sur le concept de preuve et ayant comme outil principal les mathématiques, ceci présuppose, à la base, un monde où il y a des certitudes à démontrer, des doutes qu'il faut écarter au moyen de méthodes rigoureuses, un monde régi par des lois à découvrir [1].

Le but de la recherche est alors de quantifier une vérité qui nous échappe encore et d'être capable de la démontrer par des preuves reproductibles et vérifiables [2]. Cependant, particulièrement depuis le début du $20^{\mathrm{e}}$ siècle, des critiques virulentes ont commencé à se faire entendre sur cette façon plutôt rigide de voir les choses.

G. Goodwill: Je suis personnellement tout à fait d'accord avec cette vision des choses.

L. De Groot: Je n'en doute pas, dit Lucy de Groot avec un léger sourire. Dites-moi, pensez-vous que tout sujet est étudiable par la recherche?

G. Goodwill : Oui, la recherche n'a pas et ne doit pas avoir de limite, affirma le Dr Goodwill.

L. De Groot : Parfait, je suis bien d'accord avec vous, répondit Lucy de Groot. Ne trouvez-vous pas cependant que la vision positiviste est un peu encombrante pour l'étude de certains sujets? En effet, particulièrement dans le domaine des sciences humaines et sociales, cette façon de tenter de quantifier la réalité est inefficace et souvent infructueuse [2].

G. Goodwill : Je pourrais entrevoir cette vision pour, par exemple, un sujet qui serait très peu étudié. Je vois bien que l'on pourrait à ce moment-là tenter d'explorer, de voir le sens que les gens donnent à tel ou tel phénomène ou concept, mais cela dans le but d'éventuellement bâtir de véritables hypothèses et de les mettre à l'épreuve d'une façon ou d'une autre. Là-dessus, je peux concevoir...

L. De Groot: Vous n'avez pas encore compris. Vous mettez l'un «au service» de l'autre. Moi, je vous dis que la recherche qualitative a sa place pleine et entière au sein de la science. Prenons l'exemple d'un chercheur qui voudrait étudier le concept de l'amitié. Existe-t-il un vrai concept de l'amitié, une vision plus juste que les autres, une réalité de l'amitié qui serait reproductible et fiable selon les époques, le contexte, le point de vue? Serait-ce vraiment rendre justice à cette réalité que de tenter de la quantifier, de tenter de la faire entrer dans un cadre? L'approche hypothéticodéductive que vous favorisez vous empêche d'accéder à une réalité non quantifiable et non reproductible [3].

«L'être humain construit son expérience du monde à travers une activité symbolique, c'est-à-dire que sa relation aux objets, aux personnes et aux évènements 
n'est pas directe, étant toujours médiatisée par des symboles » [4].

C'est ce que vous faites vous-même tous les jours. Sans vous en rendre compte, votre vie, votre perception du monde est construite autour des symboles. Prenons l'exemple du simple verre que vous tenez dans votre main. De façon tout à fait «objective », c'est un récipient de verre utilisé pour y mettre un liquide quelconque, mais plus souvent de l'alcool. Déjà, nous pouvons y voir une représentation socialement acquise et communément acceptée de cet objet. Vous comprendrez cependant que pour l'alcoolique qui a soif, pour le souffleur de verre qui fabrique ces objets, pour l'enfant à qui on a toujours interdit de toucher les verres plus fragiles, ce verre n'aura pas la même représentation symbolique [4].

G. Goodwill: Mais le but de la recherche est justement de se sortir de cette réalité humaine qui nous voile les yeux et d'avoir un regard neutre et objectif.

L. De Groot : Pourquoi donc ce besoin de renier cette réalité humaine qui est la nôtre? Comme l'écrivait Damasio, «que cela nous plaise ou non, tous les contenus de notre esprit sont subjectifs » [5]. Plutôt que d'essayer d'expliquer, de prouver, je vous propose plutôt de tenter de comprendre. La réalité n'est pas unique; elle est multiple, foisonnante [6].

À travers les symboles que nous construisons autour de nous, notre esprit cherche continuellement à faire du sens. Comme l'adolescent qui cherche à comprendre comment s'intégrer dans un nouveau groupe d'amis, ou comme un jeune homme qui cherche à comprendre pourquoi ses dernières relations ont été infructueuses, notre relation au monde qui nous entoure se vit à travers la recherche de sens. C'est un processus complexe, subtil, mais ô combien riche et puissant. C'est le maillon central qui définit le paradigme interprétatif ou constructiviste. Comprenez-vous maintenant la portée du changement de vision par rapport au positivisme?

G. Goodwill : Oui, je commence à voir... Je vois le but recherché, ce que vous voulez mettre en lumière, mais je dois dire que vos méthodes par contre laissent franchement à désirer.

L. De Groot: Bon, vous voilà revenu avec les «biais». Allez-y, je vous écoute...

G. Goodwill: Eh bien, laissez-moi prendre un exemple: supposons que j'adhère à votre vision et que je veuille mettre en lumière les techniques de séduction des jeunes gens dans les clubs de danse. Je questionne alors ces jeunes gens. Cependant, je suis incapable d'accéder à cette réalité que je cherche, puisque je n'ai accès qu'à ce que ces gens veulent bien me dire. Ils peuvent mentir, oublier, ne pas prendre conscience de tous les éléments! Et même dans un cas hypothétique où ils me diraient tout sans exception, je suis limité par mes propres interprétations, mes propres préjugés. Si un autre chercheur faisait la même recherche, il pourrait avoir une interprétation différente, parce que le chercheur fait partie lui-même des variables de l'expérience qualitative! La subjectivité et le manque de reproductibilité sont un réel problème de vos méthodes.
L. De Groot: Parfait. Vos questionnements sont légitimes. Parlons donc des méthodes qualitatives. Je répondrai à vos inquiétudes face à la subjectivité que ce soit du sujet ou du chercheur et nous parlerons de l'herméneutique.

Je me permets d'abord de revenir encore une fois sur la posture que vous adoptez pour élaborer vos critiques, car elles prennent naissance toujours du même noyau. «Bon nombre des prescriptions relatives à la fiabilité, à la validité et à la représentativité des données d'enquêtes qualitatives reposent sur un postulat implicite qui est questionnable, à savoir que la scientificité est fonction de la quantité d'information ou de leur contrôle précis et reproductible» [3]. Je tenterai d'ébranler cette affirmation.

D'abord, vous vous questionnez sur la subjectivité du sujet. Vous vous demandez si vos résultats seront «fiables ». Si je reprends votre exemple de séduction dans les clubs de danse, si votre objectif est bien de comprendre la manière dont les jeunes procèdent, vous pourriez, en plus de vos entrevues, aller directement sur le terrain afin d'observer les comportements. Cela enrichirait grandement votre compréhension du tout. En effet, l'observation sur le terrain, les entrevues de groupe, les entrevues individuelles font partie de «l'enquête qualitative de terrain» [4]. Cependant, je pousserais le questionnement encore plus loin en vous citant Ameigeiras :

[La compréhension n'est pas] le résultat de la «découverte» d'une qualité occulte dans l'objet de recherche que nous récupérerions grâce à notre stratégie de recueil d'information, ou que nous localiserions à partir de l'analyse d'une entrevue ou de notre participation à un événement. Et il en va ainsi car, plutôt que donner de la visibilité à «quelque chose » qui se trouve là, le sens est construit par l'acteur et reconstruit dans l'interprétation [7].

Voyez-vous, les méthodes qualitatives sont des méthodes de construction de sens, de compréhension et non pas de découverte, ni de démonstration [6].

Ensuite, par rapport au chercheur lui-même, vous soulevez le point que sa subjectivité rend les résultats invalides et fort probablement non reproductibles. Au lieu de tenter de justifier ce fait et de l'excuser à vos yeux, je commencerai par dire, au risque de vous choquer, que, à l'inverse, dans la recherche qualitative, il est tout à fait assumé et attendu que le chercheur soit l'ultime instrument [2]. Il importe donc de comprendre que l'analyse qualitative nous parle souvent «autant de l'analyste, de sa société et de sa culture que du phénomène analysé » [4].

La subjectivité a pleinement sa légitimité dans la recherche qualitative, puisque, dans le domaine des sciences sociales, tout est interprétatif. C'est la réalité même de l'expérience humaine. Toute notre compréhension du monde se fait via ce regard humain. « Nous sommes voués à l'anthropomorphisme, que nous le voulions ou pas» [2]. Vous voyez la subjectivité comme un ennemi à 
chasser mais, dites-moi, y-a-t-il, selon vous, quelque chose de plus vrai que la perception même? Vous faites des yeux ébahis, mais réfléchissez un instant. Comme Husserl l'a bien compris, «sous toutes ses formes, la connaissance est un vécu psychique. [...] La connaissance est donc bien seulement connaissance humaine, liée aux formes intellectuelles humaines, incapable d'atteindre la nature des choses mêmes, d'atteindre les choses en soi » [8]. Nous sommes prisonniers de nos perceptions. Nous ne pouvons parvenir à la connaissance que par nos sens. Comme le disait Descartes avec le «je pense, donc je suis », nos perceptions ne sontelles pas la seule certitude que nous puissions jamais avoir? ... Tout le défi repose dans la capacité, à partir de ces perceptions, à construire l'interprétation.

G. Goodwill : Mais alors, êtes-vous en train de dire que tout se vaut? Tout le monde peut faire ce qu'il veut, interpréter comme bon lui semble? C'est bien ce que vous semblez dire, puisqu'on ne peut aller à l'encontre des perceptions selon ce que vous dites. Il n'y a, à mon sens, aucune différence entre la recherche qualitative et le texte d'opinion! D'ailleurs, un autre point majeur m'est apparu en assistant à la présentation d'un de vos collègues. Il n'avait presque pas de cadre théorique! Êtes-vous à ce point au-dessus de vos prédécesseurs que vous n'avez même pas à fournir l'effort intellectuel de situer votre recherche dans un cadre adéquat?

L. De Groot : Je pense que vous m'épargnez dans vos commentaires. Je devine, dans vos propos, l'accusation sous-entendue de paresse intellectuelle, voire d'égocentrisme, n'est-ce pas?

G. Goodwill: Je ne l'aurais pas formulé ainsi, mais puisque vous le mentionnez...

L. De Groot : C'est bien. Jouons cartes sur table afin de pouvoir crever l'abcès. Je vais vous parler de ce processus de construction de l'interprétation que l'on appelle l'herméneutique. Puis, nous parlerons de rigueur de la recherche et de cadre théorique.

Commençons tout d'abord par explorer ce mystère qu'est la construction de l'interprétation dans le processus qualitatif. Ce qu'il importe de comprendre est que l'herméneutique ne constitue pas une méthode, ni une procédure à appliquer, mais plutôt «une réflexion philosophique sur le phénomène de la compréhension et le caractère interprétatif de notre expérience du monde » [9]. Ceci ne produit pas de recette, ni de protocole pour bâtir une compréhension. Cela peut alors donner l'impression que tout le monde peut faire «n'importe quoi» comme vous dites.

Vous ne comprenez pas la différence entre la recherche qualitative et le texte d'opinion. Pourtant, «l'interprétation authentique n'est pas une gageure ou une parole en l'air, même si elle peut momentanément prendre appui sur de telles conjectures » [4]. Il est vrai que le chercheur a ses préjugés ou ses pré-connaissances en débutant la recherche. Comment ne pas en avoir? Il serait absurde de chercher à les nier ou de tenter de les éliminer. Cela ne veut cependant pas dire que le chercheur peut dire ce que bon lui semble. Comme le dit bien Paillé: «L'herméneutique ne peut pas faire l'économie de la saisie phénoménologique de son objet, à savoir cette rencontre où la présence de l'objet n'est pas négligée, banalisée ou «snobée », mais est, au contraire, authentiquement reçue, attestée, honorée » [4]. Il s'agit bien de construire des interprétations qui sont valides, c'est là où repose la complexité de l'analyse qualitative. Ceci est encore plus complexe compte tenu du défi de la double herméneutique, c'est-à-dire celle du sujet et celle du chercheur [7]. Comment alors en arriver à une interprétation valide? Comme l'écrit Ameigeiras, «un type d'appréciation s'impose qui, tout en reconnaissant la pluralité de sens, explicite quelques interprétations comme plus "probables" ou "possibles" que d'autres » [7]. Illustrons ceci par un exemple : nous sommes dans un musée, devant une sculpture de pierre de forme plutôt abstraite. Je demande à chacun de tenter de mettre en lumière la signification de cette œuvre. Nous devinons que le critique d'art, le géologue, l'enfant ou le psychologue n'auront pas la même interprétation de ce qu'ils ont devant eux. Est-ce qu'une des interprétations sera plus vraie que l'autre? Devraient-ils tous tenter de mettre leurs préjugés de côté? Est-ce que cela en ferait une interprétation plus juste? Il est donc important de bien faire la différence entre posture et attitude du chercheur. La posture est intrinsèque au chercheur, ce qu'il ne peut que difficilement changer, ce qui appartient à son passé, son histoire; bref ce qui tient de ses préjugés légitimes. Son attitude tient plutôt du fait de sa façon d'aborder ce qui se trouve devant lui et qui peut changer, évoluer, etc. Celle-ci se doit, pour être juste, d'être ouverte face à la complexité qui se trouve devant elle. La posture et l'attitude sont tous les deux essentiels à la compréhension [4]. De ce fait, le rôle du chercheur ou «de l'interprète prend une dimension supplémentaire du fait que son apport est bien plus qu'une lecture d'un texte, il est une contribution au sens même de ce texte (...) » [4]. Ce n'est donc pas un processus aléatoire.

Je me permettrai de poursuivre sur ce concept de rigueur intellectuelle. Vous avez mentionné le soi-disant manque de cadre théorique de certaines recherches qualitatives. Vous soulevez le fait que toute recherche scientifique devrait être effectuée suite à une recension exhaustive des écrits pour y bâtir un cadre théorique adéquat. Ceci sous-tend que toute recherche, tout savoir scientifique devrait s'appuyer sur la théorie. Ceci ne prend cependant pas en compte le savoir expérientiel du chercheur lui-même, savoir qui est particulièrement important dans le domaine des sciences humaines et sociales [3]. «La problématique doit être, d'une certaine façon, minimale. Il ne s'agit ni de poser les bases d'une thèse (dans le sens philosophique), ni d'articuler une démonstration à des fins de vérifications mais de mettre en projet une enquête de terrain» [2]. Alors, comment trouver l'équilibre entre savoir théorique, savoir expérientiel et données d'enquêtes? C'est ce que Paillé et Mucchielli appellent l'équation intellectuelle du chercheur en expliquant :

Ce n'est pas que le théorique ou que la recension des écrits pose problème dans l'enquête qualitative; tout au contraire, leur définition trop étroite ainsi que le 
réductionnisme dont ils sont l'objet à travers un rapport figé et formalisant aux impératifs de l'enquête reviennent à leur banalisation aux moments clés de l'interprétation [4].

Le principe, que vous devez fort bien connaître, de faire d'abord une référence aux écrits, puis bâtir le cadre théorique, poser une question, faire une hypothèse, aller sur le terrain, pour enfin répondre à la question; cela ne convient pas au modèle qualitatif [4]. En effet, l'interprétation est un processus continu, un processus vivant, itératif. Le design méthodologique évolue souvent tout au long du processus, les étapes d'analyse et de collecte de données n'ont pas de frontières claires et définies [9]. La théorie doit donc nourrir le processus de compréhension et non pas le cadrer, ni le restreindre.

G. Goodwill : Mais n'avez-vous donc pas de critères permettant de juger de la méthodologie de vos pairs?

L. De Groot: Bien sûr, cela ne se fait pas sans une rigueur qui est propre à la recherche qualitative. Comme le dit Savoie-Zajc [10], certains critères méthodologiques ont été décrits par Guba et Lincoln [11,12] afin de servir de jalons aux chercheurs. On parle alors de critère de transférabilité, de crédibilité, de fiabilité et de confirmation [10]. Pour ce qui est de la crédibilité, par exemple, il s'agit de voir si l'interprétation qu'en tire le chercheur est plausible. Cela peut se faire, entre autres, par des stratégies de triangulation [10].

Cependant, je n'entrerai pas trop dans les détails et je me permettrai même d'émettre des mises en garde face à ces fameux critères afin de ne pas dévier du débat principal qui nous occupe. En effet, comme l'écrit Paillé: «la tentative de trouver des équivalents «qualitatifs» aux critères de scientificité issus de la recherche expérimentale participe, dans ces excès, de la même méprise sur la logique de l'enquête qualitative» [3]. Ces critères peuvent nous guider, mais comme je vous l'ai dit à plusieurs reprises, il faut se méfier de tout ce qui tenterait de vouloir contrôler et restreindre les méthodes qualitatives, car se serait alors retomber dans le positivisme et perdre l'essence même du paradigme interprétatif. Cela ne tient ni de la paresse, ni de raccourci intellectuel, bien au contraire! La recherche qualitative demande temps, travail, rigueur, réflexion, sensibilité et intelligence; j'espère que vous commencez à l'entrevoir!...

G. Goodwill : Je commence à saisir en effet... Si donc je voulais décrire les méthodes qualitatives en quelques mots à un collègue naïf en la matière, comment résumeriez-vous votre propos?

L. De Groot : Eh bien, je résumerais à ce collègue que la recherche et l'analyse qualitatives sont d'abord et avant tout issues du paradigme interprétatif dont elles tirent toute leur âme et leur essence. J'ajouterais que celui-ci se base, non pas sur la découverte ou la démonstration de la vérité, mais sur la construction de sens, la compréhension de phénomènes complexes pour lesquels il existe une pluralité d'interprétations possibles. La subjectivité à la fois du chercheur et du sujet ne doivent pas être vues comme une faiblesse, mais bien comme une force de la recherche qualitative, puisque son domaine d'étude est avant tout humain et donc, bâti à travers les perceptions de chacun. J'insisterais sur l'importance de la place du chercheur au cœur même de la recherche qu'il entreprend, lui qui, grâce à sa posture et son attitude, arrive à mettre en lumière une interprétation valide. C'est un processus long, ardu et rigoureux que celui de l'herméneutique, mais ô combien riche et vaste. Surtout, si j'étais à votre place, je terminerais en disant que la recherche qualitative n'a rien à envier aux méthodes quantitatives, bien au contraire. Elles ont pleinement gagné leur place au sein de la communauté scientifique!... [2].

G. Goodwill: J'en prends bonne note, répondit $D r$ Goodwill en riant. En tous cas, très chère, vous avez bien débattu et avez su gagner mon respect et mon admiration durant cet argumentaire.

L. De Groot: Qui sait, mon ami, si un jour vous ne viendrez pas grossir nos rangs de chercheurs qualitatifs!

G. Goodwill : Oh, je ne pense pas que la médecine soit encore prête à cela...

L. De Groot: Détrompez-vous, mon cher, détrompez-vous! Le milieu médical est un excellent terrain pour une chercheuse qualitative comme moi et se prête bien à la méthode qualitative [13].

G. Goodwill : Vraiment? Alors peut-être travaillerons-nous un jour sur un projet commun? Et n'ayez crainte, je ne parle pas de méthodes mixtes! répondit Dr Goodwill avec un grand sourire.

L. De Groot: Alors, c'est un rendez-vous..., lança Lucy de Groot en lançant un clin d'œil à son compagnon.

\section{Remerciements}

Je remercie Pierre Paillé qui m'a fait découvrir la beauté de la recherche qualitative et Patrice Chrétien Raymer pour son aide et son soutien.

\section{Conflits d'intérêts}

L'auteur déclare n'avoir aucun lien d'intérêt concernant les données publiées dans cet article.

\section{Approbation éthique}

Non requise.

\section{Références}

1. Busino G. La preuve dans les sciences sociales. Rev Eur Sci Soc 2003;XLI(128):1-61.

2. Paillé P. La recherche qualitative... sans gêne et sans regrets. Rech Soins Infirm 1997 (50):60-64.

3. Paillé P. La recherche qualitative: une méthodologie de la proximité, in Problèmes sociaux. Tome III. Théories et méthodologies de la recherche, Dorvil H (dir.). Québec: Presses de l'Université du Québec, 2007, p. 409-443

4. Paillé P, Mucchielli A. L'analyse qualitative en sciences humaines et sociales. $4^{\mathrm{e}}$ éd. Domont: Éditions Armand Colin, 2016. 
5. Damasio AR. Le Sentiment même de soi : corps, émotions, conscience. Paris : Odile Jacob, 1999.

6. Albert M-N, Couture M-M. La légitimation de savoirs issus de récits autobiographiques dans une épistémologie constructiviste pragmatique. Rech Qual 2013;2: 175-200.

7. Ameigeiras A. L'herméneutique dans l'approche ethnographique. Du labyrinthe de la compréhension au défi de l'interprétation. Rech Qual 2009;28:37-52.

8. Husserl E. L'Idée de la phénoménologie. Paris: Presses Universitaires de France, 1970.

9. Grondin J. Le tournant herméneutique de la phénoménologie. Paris: Presses Universitaires de France, 2003.
10. Savoie-Zajc L. La recherche qualitative/interprétative en éducation, in La recherche en éducation : ses étapes, ses approches, Karsenti T, Savoie-Zajc L (sous la direction de). Montréal: ERPI, 2011, p. 123-146.

11. Guba EG, Lincoln YS. Epistemological and methodological bases of naturalistic inquiry. Educ Technol Res Dev 1982;30:233-52.

12. Lincoln YS, Guba EG. Naturalistic inquiry. Beverly Hills (CA): Sage, 1985.

13. Pelaccia T, Paillé P. Les approches qualitatives: une invitation à l'innovation et à la découverte dans le champ de la recherche en pédagogie des sciences de la santé. Pédagogie Médicale 2009;10:293-304.

Citation de l'article : Codsi M.-P., Une rencontre épistémologique. Pédagogie Médicale, 2017:18;25-30 